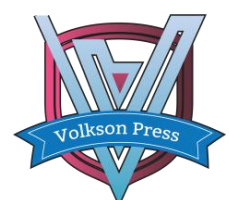

Contents List available at VOLKSON PRESS

Computer Science and Artificial Intelligence(CSAI)

DOI : http://doi.org/10.26480/iscsai.01.2017.49.51

\title{
Indoor Human Activity Recognition Method Using Csi Of Wireless Signals
}

\author{
Mohammed A. A. Al-qaness ${ }^{1 *}$, Yousif Al-Eryani2 ${ }^{2}$ Nashat Al-jallad ${ }^{2}$ \\ ${ }^{1}$ International School of Software, Wuhan University, 430079, Wuhan, China \\ ${ }^{2}$ School of Computer Science and Technology, Wuhan University of Technology, 430070, Wuhan, China \\ *Corresponding Author Email: alqaness@whu.edu.cn
}

This is an open access article distributed under the Creative Commons Attribution License, which permits unrestricted use, distribution, and reproduction in any medium, provided the original work is properly cited

\section{ARTICLE DETAILS}

\section{Article History:}

Received 02 october 2017

Accepted 06 october 2017

Available online 11 october 2017

Keywords:

Human activity recognition, CSI, Wi-Fi, Device-free
ABSTRACT

Human activity recognition has been studied for decades by leveraging vision-based and sensor-based technologies. However, the drawback of such techniques such as short-range area, and invasion of human privacy in vision-based technology, and inappropriate usage for sensor devices or inconvenient feeling of the user to carry sensor devices. All of these reasons encouraged researchers to wireless-based sensing technology or called device-free because the user needn't carry devices nor monitoring with a camera. In this regard, we present a device-free activity recognition system by exploiting the Channel State Information (CSI) of Wi-Fi signals which recognizes three dynamic activities. We build our prototype with a high efficient feature extraction algorithm and an agile and accurate classification algorithm. To examine the feasibility and performance of the proposed system, hundreds of experiments have been implemented in LOS and NLOS scenarios in a dynamic environment with different volunteer users. The experiment results show that the proposed method has gained a high-accuracy rate in both LOS and NLOS.

\section{Introduction}

Human activity recognition (HAR) has increasingly attracted intense academic and industrial interest due to its various applications in real life such as elderly monitoring. In general, traditional HAR systems considered as device-based approaches such as vision-based, body-worn sensors, and mobile-phone interior sensors [1-3]. However, all have certain drawbacks. For instance, vision-based systems cannot work through-wall and require good light condition, moreover, invasive human privacy. Sensor-based approaches are inappropriate for the user to carry a device that sometimes easy to forget or inconvenient in some situations. All of these reasons encouraged researchers to leverage wireless-based sensing technology or called device-free because the target objects needn't carry devices nor monitoring by a camera.

Therefore, recently, radio signals such as Wi-Fi signals are utilized to track human motion and activities. A group researcher introduced the concept of device-free human location detection using only a ubiquitous Wi-Fi router by leveraging the fluctuation of the received signal strength indicator (RSSI) [4]. Thereafter, many device-free RSSI-based approaches have been proposed with different scopes such as localization and motion detection and activity recognition [5,6]. However, RSSI suffers from instability and severe deterioration in complex environments. Most recent, the channel state information (CSI) of wireless PHY layer has gained more attention to be utilized for indoor positioning, motion tracking, and activity recognition [7-9]. Unlike RSSI, CSI is measured from radio link per OFDM subcarrier of each received packet. Therefore, CSI has more information since RSSI value is only per received packet. Moreover, CSI is more robust to complex environments.

In this paper, we present a device-free activity recognition system by leveraging the prosperities of channel state information (CSI) of wireless signals such as Wi-Fi. We use only an access point (TP-Link) and a detection point (Lenovo laptop). The target user is asked to implement activities without carrying any wireless devices nor monitored by a camera. We extract the CSI information, filter out the noise, extract the useful features, and then apply a machine learning algorithm to classify the proposed activity.

Our main contributions are as follows:
We present an efficient method to extract the useful CSI information from $\mathrm{Wi}-\mathrm{Fi}$ signals in order to reduce the multidimensional data. Then, we design a low-pass filter to remove the electromagnetic noise.

- $\quad$ A Principal Component Analysis (PCA) is applied to reduce CSI dimensionality and to select sensitive CSI streams that have more fluctuation due to human motion in the test area. Then a band of features extracted to satisfy classification process.

- Support Vector Machine (SVM) is applied to classify the proposed activities. The classification algorithm has achieved a high accuracy in both LOS and NLOS scenarios.

The remainder of this paper is organized as follows. The methodology is described in the next section. In Section 3, we describe the evaluation experiment setting and results. Lastly, we conclude the paper in section 4 .

\section{METHODOLOGY}

In this section, we explain the architecture of our system and the methodology. Figure 1 shows the workflow of the proposed method. First, the system extracts CSI from Wi-Fi signals that reflect the human motion to the receiver. Then, our system preprocesses the collected signals by modeling signals as stream vectors and removes the noise. After that, PCA algorithm is applied to reduce the dimensionality of the CSI streams. Then, our system extracts a band of features labeled as an input vector of the classification algorithm. In what follows, we describe each process in details.

\subsection{CSI Data Collection and Preprocessing}

The proposed system collects radio signals from the fixed access point in the test environment. Let $R$ be the received signal and $T$ is the transmitted signal. The received signal can be expressed as:

$$
R=H \cdot T+N
$$

where $H$ is the channel state information, $i, j$ are the streams and subcarriers, respectively? $N$ is the electromagnetic noise. CSI can be represented as Equation 2: 


$$
H_{i, j}=\left\|H_{i, j}\left(f_{k}\right)\right\| e^{\angle H_{i, j}\left(f_{k}\right)}
$$

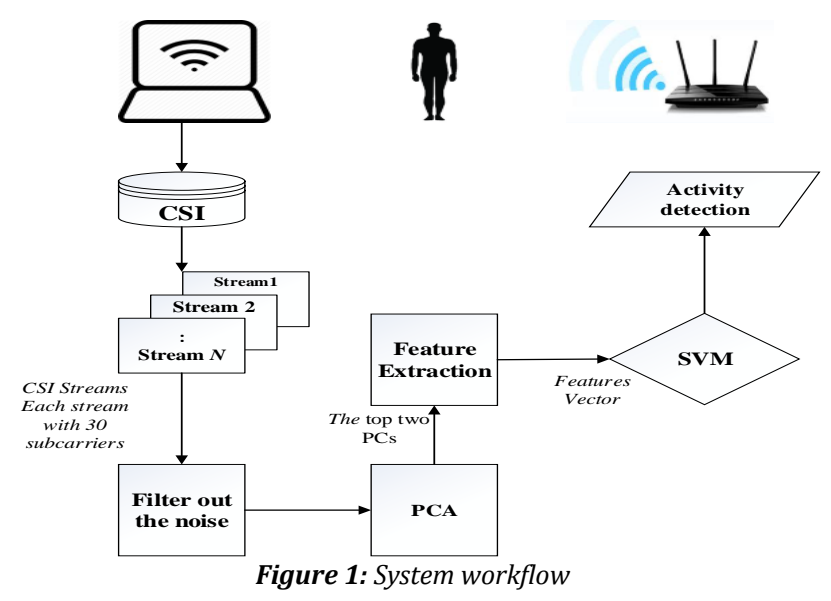

where $\left\|H_{i, j}\left(f_{k}\right)\right\|$ is the amplitude and $\angle H_{i, j}\left(f_{k}\right)$ is the phase. The measurement of CSI depends on the amplitude and phase of CSI at a time point. If we have a number of transmitter antennas $N_{t}$ and receiver antennas $N r$, the CSI of each time point is measured on 30 OFDM subcarriers for the receiver of WLAN (802.11). Therefore, the CSI measurements are $N_{t^{\times}} N_{r^{\times 30}} . N_{t^{\times}} N_{r}$ is the number of CSI streams, each stream contains 30 subcarriers. In our implementation, the transmitter is a Wi-Fi router equipped with three transmitter antenna, and the receiver is a laptop equipped with IWL5300 with three antennas. Thus, CSI can be described as Equation 3:

$\left\|H_{i, j}\left(f_{k}\right)\right\|=\left[\begin{array}{ccc}\left\|H_{1,1}\left(f_{k}\right)\right\| & \ldots & \left\|H_{1,30}\left(f_{k}\right)\right\| \\ : & : & : \\ \left\|H_{9,1}\left(f_{k}\right)\right\| & \ldots & \left\|H_{9,30}\left(f_{k}\right)\right\|\end{array}\right]+N$

Many methods can be applied to represent the collected CSI data. Here, to reduce computational complexity and reduce data dimensions, we use the mean of the 30 subcarriers of each stream as a vector that represents CSI. So, in our system CSI is measured as nine streams. Figure 2 plots CSI of a fall experiment, as shown in Figure 2 (a) the collected data still has noise from the neighboring radio devices. Therefore, we design an exponential filter to remove out the noise and to obtain the real trends of CSI streams that caused because human activity. Figure 2 (b) shows CSI streams after filtering. The figure shows that our filter is effective and removed the whole noise.

\subsection{Feature Extraction}

The fluctuation of CSI measurements caused by human motion occurs across all the streams and subcarriers in the wireless channel. The different streams have a distinctive sensitivity to the change of CSI due to human movement as shown in Figure 2. To capture the dominant fluctuation of CSI caused by human activity motion, we apply Principal Component Analysis (PCA) across all the streams of the raw CSI that collected from IWL 5300 NIC and filtered out by a low-pass filter. After that, we select the top two principal components. Then we extract the following features: mean, median, standard deviation, interquartile range, second central moment, Third central moment, skewness, kurtosis, dominant frequency, and entropy. Finally, we concentrate the ten features values that extracted from the top two PCs into a single feature vector with the dimensionality of 20 .
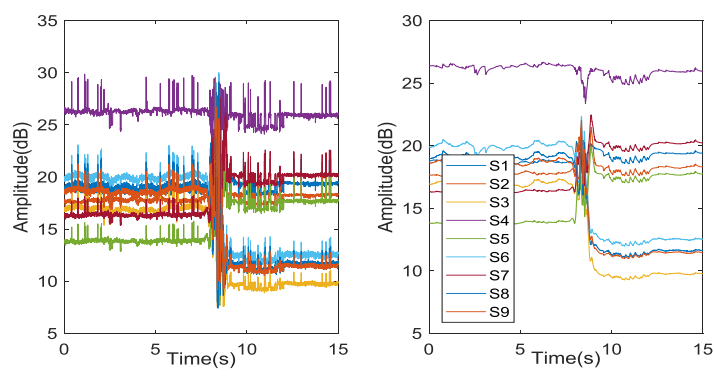

Figure 2: (a) Raw CSI amplitude before filtering. (b) Raw CSI after lowpass filter

\subsection{Activity Classification}

We adopt the well-known Support Vector machine (SVM) algorithms to classify the proposed human activities with the Gaussian radial basis function (RBF) kernel which non-linearly maps samples into a higher dimensional space. The radial basis function can be defined as:

$$
K\left(x_{i}, y_{i}\right)=\exp \left(-\gamma\left\|x_{i}-y_{i}\right\|^{2}\right), \gamma>0
$$

where $x, \mathrm{y}$ and $\gamma$ are the training, label and kernel parameter, respectively. The open source libSVM (Chang \& Lin 2011) is used to implement SVM with the Gaussian radial basis function (RBF).

\section{EVALUATION}

\subsection{Experiment Setup}

Many experiments have been conducted in two different environments, a Lab room with a size of $23 * 23 \mathrm{ft}$. and an apartment with two bedrooms and a living room with a total size of $40^{*} 40 \mathrm{ft}$. Moreover, five volunteer users conducted the three supposed activities individually. For equipment that we use in our experiments, we only use one Wi-Fi router with three antennas as a transmitter, and the receiver is a laptop with IWL5330 NIC and installed the open source CSI-TOOLS [10]. We implement the proposed activity in two scenarios as follows:

1- LOS scenario: the target user, transmitter, and receiver all placed in the same room. The target user implemented activities between the transmitter and receiver.

2- NLOS: the user and the receiver are placed in the same room, where the transmitter is placed in adjacent room separated with one wall.

\section{$3.2 \quad$ RESULTS}

We collected many samples of each activity from five users divided into five groups. Thereafter, we build our classifier in each group with 100 samples of each activity, and we measure the 5-cross validation accuracy [11]. Figure 3 plots the confusion matrix of the experiment results in both LOS and NLOS scenarios. Each raw represents an actual class, where each column represents a predicted class. From confusion matrices shown in Figure 3, the averaged accuracy is $96.667 \%$ in LOS and $93.66 \%$ in NLOS.

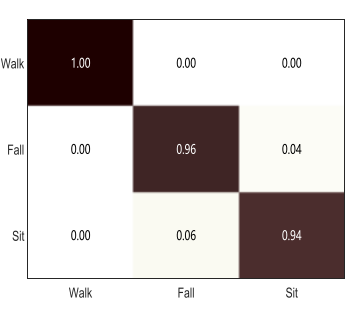

(a)

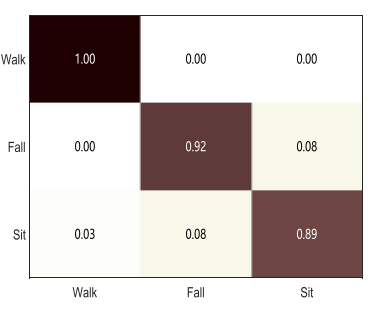

(b)
Figure 3: (a) Confusion matrix in LOS scenario. (b) Confusion matrix in NLOS scenario

Moreover, to evaluate the performance of the proposed system, precision, recall, and F1 score are used to analysis results of the experiments. Precision is the positive predictive value, Recall is the sensitivity, and F1 score or the F-measure is the weighted average of both the precision and the recall. These three mathematics rules are used as expressed in the following expressions. 
precision $=\frac{T P}{T P+F P}$

recall $=\frac{T P}{T P+F N}$

$$
F 1=2 \times \frac{\text { precision } \times \text { recall }}{\text { precision }+ \text { recall }}
$$

where $T P, F P$, and $F N$ are the true positive, false positive, and false negative, respectively. In Figure 4 we see the overall values of the precision, recall and F1 of the three proposed activities in LOS, and NLOS scenarios.

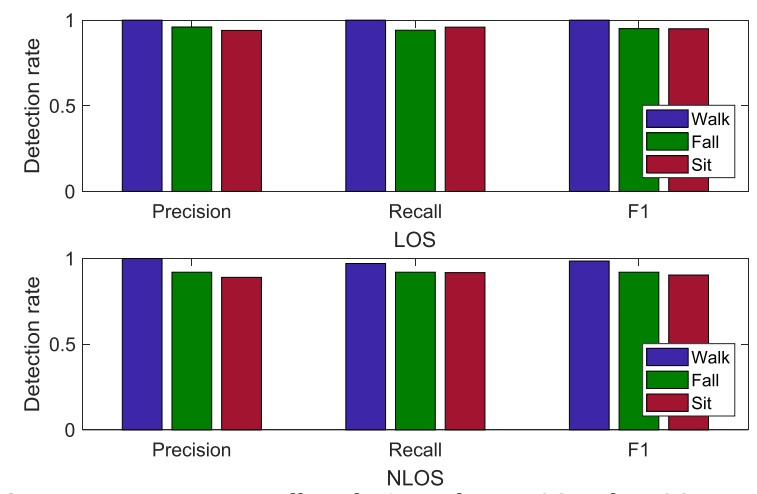

Figure 4: Precision, Recall, and F1 results in LOS and NLOS scenarios

Moreover, we compare our classification method to SVM without PCA algorithm, and k-nearest neighbor (KNN). As shown in Figure 5, our PCASVM method outperforms SVM and KNN. From Figure 5 we also notice that the accuracy increased as the number of features increased.

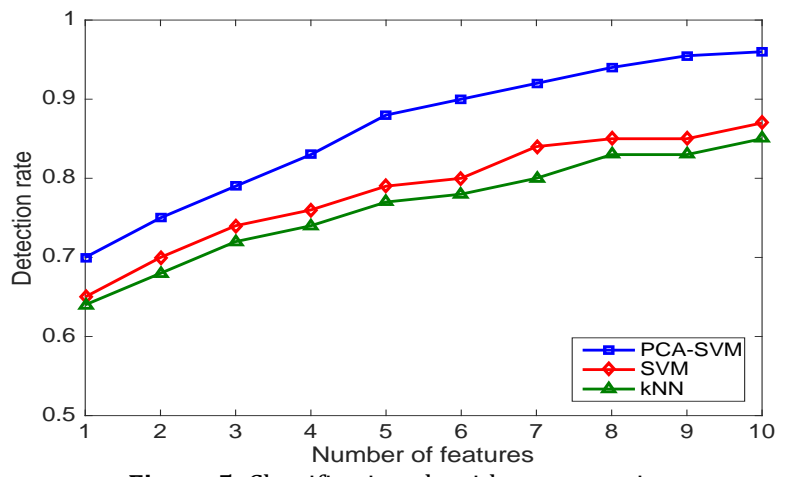

Figure 5: Classification algorithms comparison

\section{CONCLUSION}

The paper presented an indoor activity recognition method based on Channel State Information (CSI) of wireless signals. The proposed method extracts amplitude information of CSI from ubiquitous Wi-Fi router. A low- pass filter has been designed to filter out the noise caused by the surrounding electromagnetic in order to obtain the real trend of CSI that caused by human movements. PCA was applied across CSI streams to reduce the dimensionality and SVM algorithm applied to recognize the proposed activities. The proposed method has been evaluated in different scenarios, and the results approved the validity of the method. Device-free $\mathrm{Wi}$-Fi based sensing mechanism is the future of sensing technology, thus, it needs more and more investigation to address more complicated scenarios.

\section{REFERENCES}

[1] Gaglio, S., Re, G. L., Morana, M. 2015. Human activity recognition process using 3-D posture data. IEEE Transactions on Human-Machine Systems, 14 (5), 586-597.

[2] Field, M., Stirling, D., Pan, Z., Ros, M., Naghdy, F. 2015. Recognizing human motions through mixture modeling of inertial data. Pattern Recognition, 48 (8), 2394-2406.

[3] Kwon, Y., Kang, K., Bae, C. 2014. Unsupervised learning for human activity recognition using smartphone sensors. Expert Systems with Applications, 41 (14), 6067-6074.

[4] Youssef, M., Mah, M., Agrawala, A. 2007. Challenges: device-free passive localization for wireless environments. In Proc. of the 13th annual ACM international conference on Mobile computing and networking, Monteral, September, 9 (14), 222-229.

[5] Zhao, Y., Patwari, N. 2015. Robust Estimators for Variance-Based Device-Free Localization and Tracking. IEEE Transactions on Mobile Computing, 14 (10), 2116-2129.

[6] Gu, Y., Quan, L., Ren, F. 2014. Wifi-assisted human activity recognition. In Proc of IEEE Asia Pacific Conference on in Wireless and Mobile, Pali, 2830 August, 60-65.

[7] Xiao, J., Wu, K., Yi, Y., Ni, L. M. 2012. FIFS: Fine-grained indoor fingerprinting system. In Proc. of 21st International Conference on Computer Communications and Networks (ICCCN), Munich, July 30 to August 2, 1-7.

[8] Zeng, Y., Pathak, P. H., Xu, C., Mohapatra, P. 2014. Your ap knows how you move: fine-grained device motion recognition through wifi. In Proc. of the 1st ACM workshop on Hot topics in wireless, Maui, Hawai, September, $11(2), 49-54$.

[9] Al-Qaness, M.A.A., Li, F., Ma, X., Zhang, Y., Liu, G. 2016. Device-Free Indoor Activity Recognition System. Applied Sciences, 6 (11), 329.

[10] Halperin, D., Hu, W., Sheth, A., Wetherall, D. 2011. Tool release: gathering $802.11 \mathrm{n}$ traces with channel state information. ACM SIGCOMM Computer Communication Review, 41 (1), 53-53

[11] Chang, C.C., Lin, C. J. 2011. LIBSVM: a library for support vector machines. ACM Transactions on Intelligent Systems and Technology (TIST), 2 (3): 27. Software available at http://www.csie.ntu.edu.tw/ cjlin/libsvm 